A N N A L E S Annales de Bretagne et des Pays de l'Ouest

\title{
Zadora-Rio, Élizabeth (dir.), Des paroisses de Touraine aux communes d'Indre-et-Loire : la formation des territoires
}

\section{Anne Lunven}

\section{(2) OpenEdition Journals}

Édition électronique

URL : http://journals.openedition.org/abpo/1954

DOI : 10.4000/abpo.1954

ISSN : 2108-6443

Éditeur

Presses universitaires de Rennes

Édition imprimée

Date de publication : 10 avril 2011

Pagination : 195-197

ISBN : 978-2-75351410-2

ISSN : 0399-0826

Référence électronique

Anne Lunven, «Zadora-Rio, Élizabeth (dir.), Des paroisses de Touraine aux communes d'Indre-et-Loire : la formation des territoires », Annales de Bretagne et des Pays de l'Ouest [En ligne], 118-1 | 2011, mis en ligne le 09 mai 2011, consulté le 23 septembre 2020. URL : http://journals.openedition.org/abpo/1954 ; DOI : https://doi.org/10.4000/abpo.1954 
ZADORA-RIO, Élizabeth (dir.), Des paroisses de Touraine aux communes d'Indre-et-Loire : la formation des territoires, Tours, Supplément à la RACF n ${ }^{34}$, FÉRAFC, 2008, 303 p., $33 €$.

Pendant longtemps, on considéra que la paroisse était apparue dès l'époque mérovingienne, le réseau paroissial se densifiant progressivement et ses mailles se resserrant tout au long du Moyen Âge pour donner naissance aux communes actuelles. L'ouvrage collectif, dirigé par l'archéologue médiéviste Élisabeth ZadoraRio et édité dans les suppléments de la Revue archéologique du Centre de la France, remet totalement en cause ce modèle de développement paroissial et l'apparition $\mathrm{d}^{\prime} u n$ découpage territorial dès le $\mathrm{vl}^{\mathrm{e}}$ siècle. Prolongeant la réflexion amorcée dans le numéro 49 de la revue Médiévales qu'elle avait codirigée avec Dominique logna-Prat en 2005, É. Zadora-Rio tente ici de suivre, avec la collaboration de cinq autres chercheurs, la naissance de la paroisse en tant que territoire dans le cadre du diocèse de Tours ainsi que d'identifier les logiques spatiales en œuvre dans la constitution des territoires paroissiaux.

La première partie de l'ouvrage est consacrée à l'étude de l'évolution du maillage ecclésial entre le $\mathrm{vl}^{\mathrm{e}}$ siècle et la fin du Moyen Âge, à partir de la documentation écrite. Afin de pondérer le biais des sources et le décalage chronologique qui peut exister entre la fondation d'une église et sa mention écrite, une large place est également accordée à l'étude des vocables. Si É. Zadora-Rio mesure bien les risques d'une telle méthode, elle s'abstient d'utiliser les chronotypologies préétablies dont la fiabilité demeure hypothétique, pour constituer un référentiel des cultes en vogue au haut Moyen Âge, propre au diocèse, à partir des écrits de Grégoire de Tours. L'auteur conclut à la densité des lieux de culte dès cette période et réévalue à la baisse la part des fondations des $\mathrm{Xl}^{\mathrm{e}}$-x॥ ${ }^{\mathrm{e}}$ siècles, induites habituellement par la révélation documentaire qui accompagne la Réforme grégorienne. Le maillage des églises paroissiales semble ensuite se stabiliser rapidement, autour des années 1200 . L'étude de la terminologie des églises montre toutefois que le terme parochia se diffuse tardivement dans la région, à partir de la fin du $x^{\mathrm{e}}$ siècle et qu'il n'acquiert véritablement un sens spatial qu'au x\|e siècle.

Dans la deuxième partie, dédiée au système de peuplement et à la formation des territoires, É. Zadora-Rio rappelle que l'occupation du sol et l'implantation des lieux de culte sont en grande partie indépendants. Elle montre toutefois que la position hiérarchique de I'habitat au haut Moyen Âge a souvent conditionné l'accès au statut paroissial après le $x^{e}$ siècle. En revanche, les fondations castrales des $\mathrm{x}^{\mathrm{e}}$-xII ${ }^{\mathrm{e}}$ siècles ont exercé très peu d'influence sur le réseau paroissial, preuve indirecte de l'ancienneté du maillage ecclésial à l'origine des paroisses. Concernant les territoires paroissiaux, l'auteur fixe leur formation à partir des $\mathrm{x}^{\mathrm{e}}-\mathrm{xl}^{\mathrm{e}}$ siècles, lorsque des « enveloppes territoriales » se forment autour du pôle ecclésial (ensemble églisecimetière), donnant naissance à un maillage continu de l'espace sous l'effet de leur concurrence mutuelle. D'après l'auteur, ce processus est indissociable de l'évolution ecclésiologique à l'origine de la spatialisation du sacré sur terre, dans l'église et le cimetière, mise en lumière par D. logna-Prat et Michel Lauwers. D'importantes évolutions institutionnelles dans le cadre de la Réforme grégorienne vont également contraindre le fidèle à fréquenter la même église, à se faire inhumer dans son cimetière et à lui verser la dîme, contribuant à former un lien unilatéral et pérenne avec elle. La délimitation précise des paroisses est en revanche plus tardive et É. ZadoraRio emprunte aux géographes la notion de cospatialité des territoires pour souligner la superposition de réalités spatiales complexes plutôt que la juxtaposition et le parfait emboîtement des mailles formant le réseau paroissial.

Dans la troisième partie, due à I'historien moderniste Jean-Michel Gorry, le dépouillement exhaustif des procès-verbaux de délimitation qui accompagnent la 
création des communes (1790) et la constitution du cadastre par culture (1802) puis du cadastre parcellaire (1807), atteste de l'inexistence de délimitation continue des territoires paroissiaux jusqu'à la Révolution. Des espaces indivis dont l'organisation paroissiale était demeurée incertaine, subsistent même jusqu'au XIX ${ }^{e}$ siècle tels que les Landes de Saint-Martin, finalement partagées entre les sept communes limitrophes. Par ailleurs, l'auteur met en garde le lecteur contre la confusion entre paroisse et commune : ce sont les collectes, c'est-à-dire les communautés d'habitants sur lesquelles pesait l'impôt, qui ont donné naissance aux communes et non les paroisses bien que, dans les faits, la grande majorité des collectes de Touraine aient correspondu à des paroisses sous I'Ancien Régime. La filiation n'est pas non plus directe entre les communes actuelles et celles de la fin du xvIII siècle : l'étude précise de l'évolution de la carte communale entre 1790 et 2000 révèle plusieurs disparitions de communes, une création, mais aussi des échanges et des transferts de territoires, notamment pour supprimer les enclaves qui étaient nombreuses.

La restitution du réseau communal de 1790 par J.-M. Gorry qui constitue donc l'état le plus proche du réseau paroissial sous l'Ancien Régime, permet dans une quatrième et dernière partie de mener, à partir de la confection d'un Système d'Information Géographique (SIG), une étude systématique de la forme des territoires paroissiaux. É. Zadora-Rio rappelle au préalable les axiomes traditionnellement invoqués pour expliquer leur variabilité : en premier lieu, un facteur historique, selon l'idée que plus une paroisse est ancienne, plus son territoire est important et doté de frontières homogènes tandis que les créations plus récentes y auraient été découpées à l'emporte-pièce; en second lieu, des facteurs environnementaux d'après le postulat qu'une paroisse constitue le territoire vivrier d'une communauté dont la forme et la superficie sont contraintes à la fois par l'accès à des ressources suffisantes, la distance et les obstacles naturels (relief et cours d'eau). La vérification de telles hypothèses a nécessité le concours de chercheurs issus d'horizons très variés : le géographe Eric Blin a travaillé sur le rôle du réseau hydrographique dans la définition des limites communales, la pédologue Dominique Boutin a établi une classification des sols de Touraine selon leur potentiel agricole pour des sociétés préindustrielles, le statisticien Pascal Chareille a tenté d'analyser statistiquement le semis des églises et la forme des territoires paroissiaux, enfin l'archéologue Xavier Rodier a proposé une modélisation des territoires paroissiaux selon la distance au chef-lieu. Les principaux apports de cette étude, très largement expérimentale, sont de souligner l'importance des cours d'eau dans la définition des limites paroissiales. La pédologie ne semble pas intervenir dans la taille des paroisses bien qu'elle puisse très localement expliquer leur forme. Plus une église est ancienne plus son territoire paroissial est important; en revanche, un tel lien n'est pas établi concernant le tracé plus ou moins régulier de ses limites. Enfin, la distance au chef-lieu, en prenant en compte les contraintes du relief et du réseau hydrographique, explique le mieux la superficie des territoires paroissiaux, mais non leur forme. L'application de ce modèle aux semis des églises carolingiennes montre que dès cette époque un maillage continu de l'espace existait, seules les zones forestières demeurant à l'écart.

Cette étude présente des aspects particulièrement novateurs à la fois par l'étendue chronologique qu'elle couvre - du vi $\mathrm{e}^{\mathrm{e}}$ siècle à nos jours - et par l'utilisation d'un SIG, outil plus familier aux archéologues qu'aux historiens, bien que les sources archéologiques ne soient finalement que très peu sollicitées. On apprécie également l'abondance des illustrations, composées de cartes réalisées par Corinne RupinScheid et de nombreux tableaux récapitulatifs des données, qui facilitent grandement la lecture, particulièrement dans la dernière partie, très technique, dont on pouvait craindre l'aspect rébarbatif. La qualité de cet ouvrage tient notamment au fait qu'il établit clairement l'absence de délimitation précise des paroisses jusqu'à 
une époque très avancée et alerte le chercheur contre l'utilisation sans précaution du réseau communal actuel pour remonter à celui des paroisses de l'Ancien Régime. En outre, il met définitivement un terme à l'hypothèse d'un démembrement croissant du diocèse en de plus en plus petites paroisses. En revanche, le poids des héritages pour expliquer la formation des territoires paroissiaux nous semble un peu sous-évalué par É. Zadora-Rio. Certes, il est sans doute impossible de reconstituer des unités territoriales antérieures au $x^{\mathrm{e}}$ siècle à partir du réseau paroissial, mais on peut penser que des communautés ecclésiales préexistaient aux paroisses et inscrivaient déjà dans l'espace des pratiques cultuelles et sociales. Cet ouvrage très neuf marque un véritable tournant historiographique concernant l'approche de la paroisse et plus largement, la représentation et l'organisation de l'espace au Moyen Âge.

Anne LUNVEN

Buono, Alessandro, Esercito, istituzioni, territorio. Alloggiamenti militari e "case herme " nello Stato di Milano (secoli XVI e XVII), Florence, Firenze University Press, 2009, $354 \mathrm{p}$.

L'histoire militaire de l'époque moderne, quoique souvent circonscrite aux $x V^{e}$ et $\mathrm{XVII}$ siècles, connaît depuis quelques années en Italie un renouveau et un dynamisme indéniables. En témoigne ce livre récent d'A. Buono, jeune chercheur de I'Università Statale de Milan, ouvrage directement issu de sa thèse de doctorat.

S'inscrivant pleinement dans les problématiques actuelles d'une histoire militaire italienne qui se veut également institutionnelle, territoriale et sociale, A. Buono s'interroge sur l'impact de l'armée espagnole dans le duché de Milan aux xvI et $\mathrm{XVII}^{\mathrm{e}}$ siècles. Dans cette optique, le logement militaire, centre de son étude, constitue un " observatoire privilégié » pour appréhender les conséquences de la guerre et de la présence de l'armée sur les institutions locales, leurs finances, leur fonctionnement, ainsi que sur la vie quotidienne des habitants.

L'auteur s'appuie sur des sources variées. En premier lieu, les abondantes archives de l'État de Milan, utilement complétées par de non moins riches fonds communaux (Milan, Pavie et Vigevano pour l'essentiel). Le recours aux archives de la monarchie espagnole conservées à Simancas permet de mieux cerner la politique du pouvoir central. Quant à l'abondante bibliographie, elle offre un panorama de la recherche italienne récente en histoire militaire moderne et, pour d'indispensables mises en perspective, intègre nombre d'études anglo-saxonnes, françaises et espagnoles, sans négliger d'autres sciences humaines (les théories de P. Bourdieu sur le pouvoir inspirent particulièrement l'auteur).

L'étude commence avec le passage de l'État de Milan de la domination des Sforza, continuateurs des Visconti, à celle des Espagnols en 1535, et s'achève dans les années 1650, juste avant la paix des Pyrénées. Sous la domination de Madrid, I'histoire du logement militaire dans le Milanais est faite de constantes expérimentations et d'un certain nombre d'échecs principalement dus au caractère presque incessant des guerres.

Le premier chapitre de l'ouvrage examine la progressive rationalisation du logement des gens de guerre, qui s'accompagne d'une "privatisation » de leur gestion. Les Espagnols ne partent pas de rien : une législation et des administrateurs dévolus aux affaires militaires existaient déjà sous les Sforza et la nouvelle administration se développe sur ce substrat. Après la paix de 1559, 5500 à 6000 soldats stationnent 